\title{
Table-ronde de conclusion
}

Gilbert-Robert Delahaye, Fanny Hamonic, Lise Leroux, François HéberSuffrin et Christian Sapin

\section{OpenEdition}

\section{Journals}

Édition électronique

URL : https://journals.openedition.org/cel/19237

DOI : $10.4000 /$ cel. 19237

ISSN : 2262-208X

Éditeur

École du Louvre

\section{Référence électronique}

Gilbert-Robert Delahaye, Fanny Hamonic, Lise Leroux, François Héber-Suffrin et Christian Sapin,

«Table-ronde de conclusion », Les Cahiers de l'École du Louvre [En ligne], 17 | 2021, mis en ligne le 18 novembre 2021, consulté le 13 décembre 2021. URL : http://journals.openedition.org/cel/19237 ; DOI : https://doi.org/10.4000/cel.19237

Ce document a été généré automatiquement le 13 décembre 2021.

\section{(c) $(1)$}

Les Cahiers de l'École du Louvre sont mis à disposition selon les termes de la licence Creative Commons Attribution - Pas d'Utilisation Commerciale - Pas de Modification 4.0 International. 


\title{
Table-ronde de conclusion
}

\author{
Gilbert-Robert Delahaye, Fanny Hamonic, Lise Leroux, François Héber- \\ Suffrin et Christian Sapin
}

1

2

\section{Christian Sapin}

Nous avons pensé que, plutôt qu'une seule conclusion, il était intéressant de proposer cette table ronde permettant de réunir plusieurs participants du colloque SATHMA qui s'est tenu les 24 et 25 Septembre 2020 à l'École du Louvre et dont ont été tirées les contributions précédentes. Fanny Hamonic, conservatrice au Musée d'archéologie nationale, Lise Leroux ingénieur de recherche au Laboratoire de recherche des monuments historiques, Gilbert-Robert Delahaye, président honoraire de plusieurs sociétés d'érudition et François Héber-Suffrin, maître de conférences émérite des universités, apporteront ainsi leurs contributions en contrepoint à ce qui a été dit précédemment

\section{Gilbert-Robert Delahaye}

Tout d'abord, j'adresse mes compliments et mes remerciements aux auteurs des communications qui durant ces deux jours, nous ont informés de l'état d'avancement des travaux en cours dans les différentes directions de recherche relatives à la sculpture tardo-antique et haut-médiévale. Merci aussi aux représentants d'autres pays qui nous ont brossé un panorama de ces recherches dans leurs pays respectifs, recherches souvent plus avancées qu'en France et qui ouvrent des perspectives aux chercheurs français.

4 Ayant le discutable privilège de compter aujourd'hui parmi les plus âgés et les plus anciens chercheurs qui se vouent à l'étude du haut Moyen Âge, j'ai, bien sûr, connu les initiatrices des recherches sur les monuments sculptés du $\mathrm{IV}^{\mathrm{e}}$ au X $\mathrm{X}^{\mathrm{e}}$ siècle, Mesdames Denise Fossard, May Vieillard-Troiekouroff et Élisabeth Chatel. Je conserve le souvenir ému de conversations avec May Vieillard-Troiekouroff et son mari. Même si les recherches de ces pionnières ont parfois été accueillies avec réserve, en raison notamment du cadre d'étude (par département géographique du lieu de conservation des œuvres), leur choix s'est avéré par la suite éminemment utile. Puisque j'évoque ces 
trois éminentes précurseuses, ayons garde de ne pas oublier leurs collaborateurs rétribués ou bénévoles, telle Madame Denise Fourmont, photographe au CNRS, qui, chaque année, partait en mission avec l'une de ces dames pour recueillir l'image des monuments sculptés tardo-antiques et haut-médiévaux d'un département français. Les tirages photographiques qui en ont résulté sont aujourd'hui la richesse des archives que Messieurs Pierre-Yves Le Pogam et Vincent Rousseau ont évoquées. Un autre de leurs collaborateurs mérite aussi qu'on évoque ici son souvenir et son œuvre, c'est Pierre Rousseau, leur dessinateur, chercheur lui-même, dont l'érudition n'avait d'égale que la modestie et le talent. Illustrateur du volume que la Marquise de Maillé consacra aux Cryptes de Jouarre, publié en 1971, il a laissé sur ce monument et les trésors d'art qu'il recèle un portefeuille de plusieurs dizaines de dessins, dont bon nombre sont demeurés inédits (fig. 1-3).

Fig. 1. Cryptes de Jouarre

(RYPTES DE JOUARRE

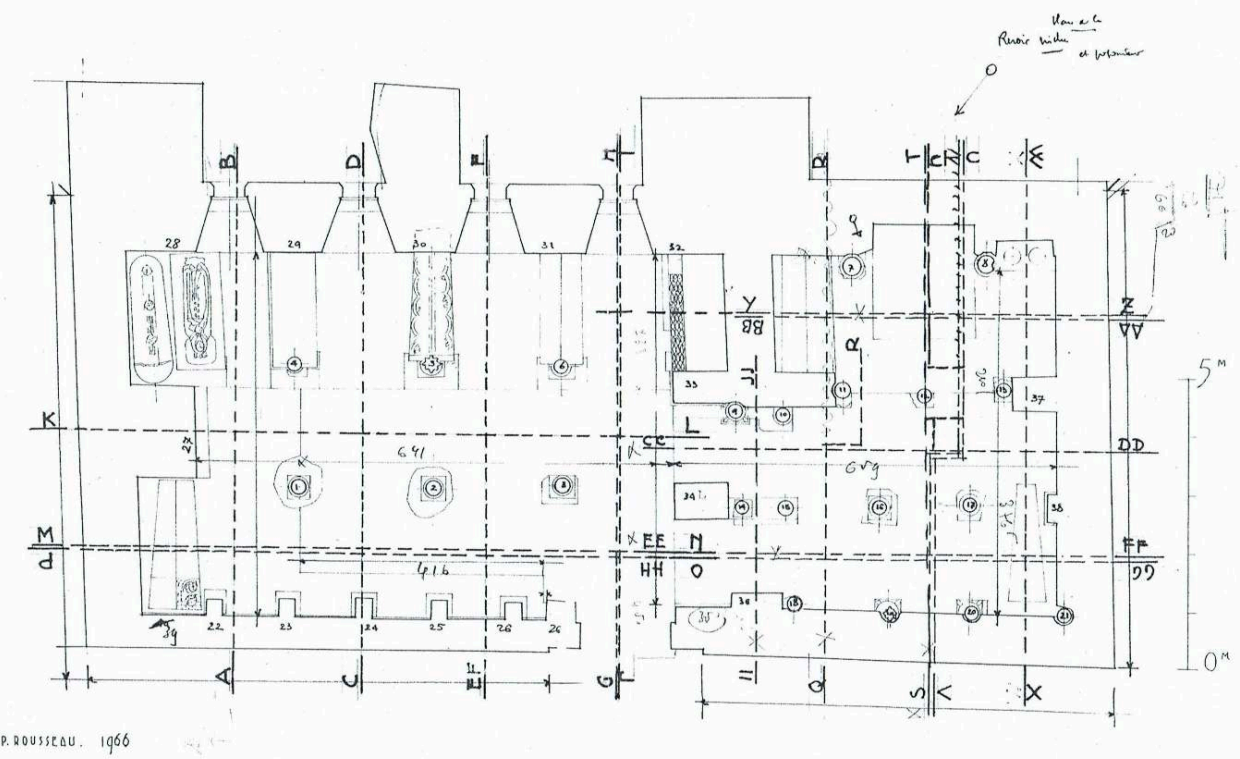

Sur le plan des cryptes de Jouarre (levé par Pierre Rousseau), disposition des coupes qu'il exécuta. Ce document de travail (mis au net dans le livre Les cryptes de Jouarre) reflète bien la préparation d'une œuvre personnelle et non pas la copie d'éventuels relevés de l'abbé Thiercelin.

(c) Collection G.-R. Delahaye 
Fig. 2. Crypte Saint-Paul de l'Abbaye de Jouarre

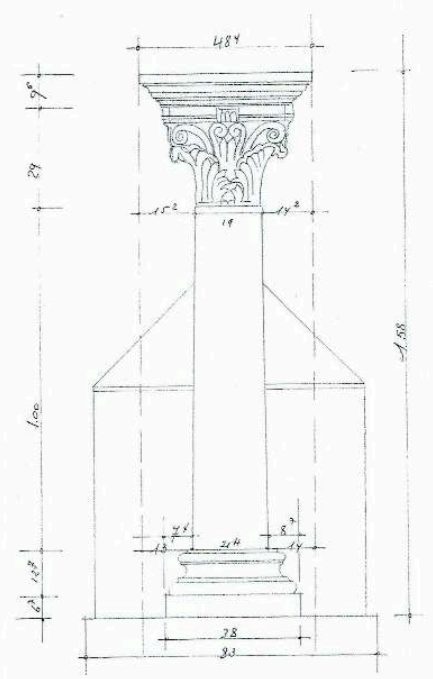

FACE OUEST

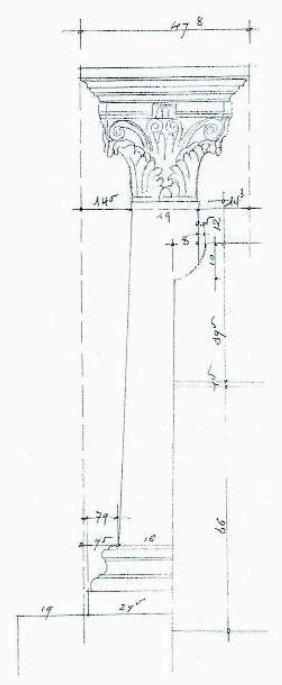

FACE SUD

SOLOMNE 6

ABBAYEDE JOUARRE IRYPTE SAINT-PAUL

P.R. 1967

Relevé par Pierre Rousseau, de la colonne 6 de la crypte Saint-Paul, engagée dans le cénatophe de Mode.

(c) Collection G.-R. Delahaye

Fig. 3. Tailloirs \& impostes
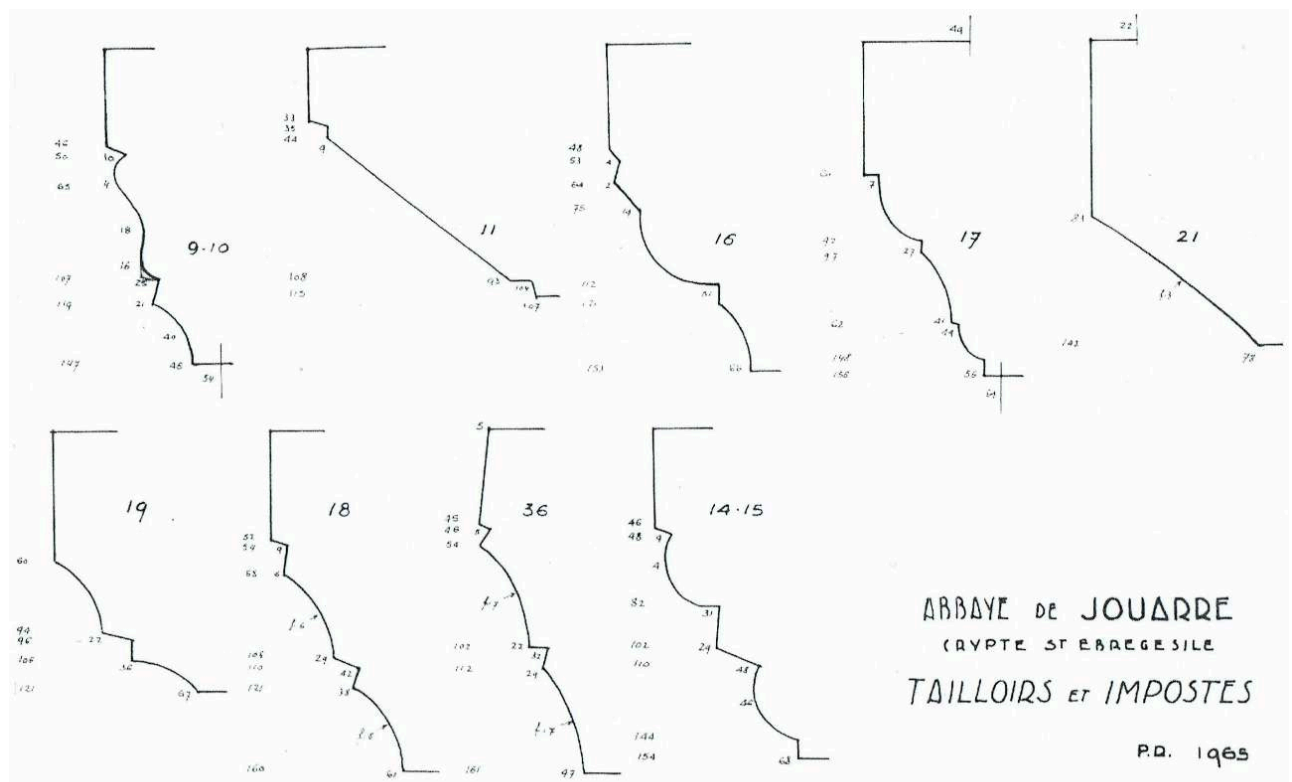

ABBAYE DE JOU $\triangle R R E$

TAILLOIRS ET IMPOSTES

P.D. 1965

Profils des tailloirs des chapiteaux et des impostes de la crypte Saint-Ébrégisile de Jouarre, relevés par Pierre Rousseau.

(c) Collection G.-R. Delahaye 
5 Et puis, au-delà des fidèles soutiens de ces trois pionnières, on ne peut passer sous silence les noms de quelques-uns de leurs « patrons » de recherche : André Grabar, qui avait initialement réuni ces trois femmes autour de l'art chrétien oriental, avant qu'elles ne s'attachent à la prolongation des recherches que Jean Hubert avait initiées dans son ouvrage L'art pré-roman.

Fig. 4. Page de titre, L'Art pré-roman

LES MONUMENTS DATES DE LA FRANCE COLLECTION PUBLIEE SOUS LA DIRECTION DE M. LOUIS HAUTECEEUR

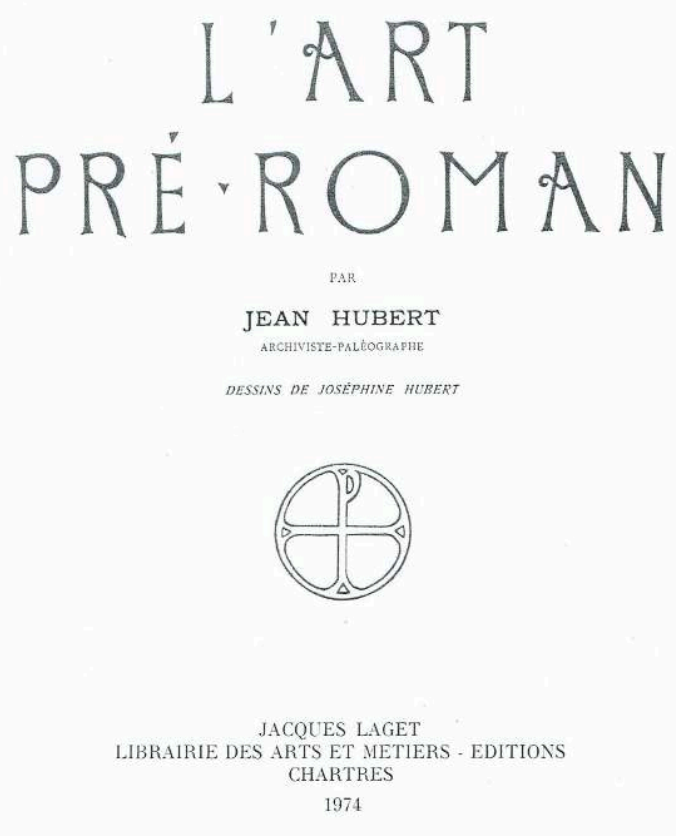

Ouvrage de Jean Hubert publié en 1974 aux éditions Chartres. 
Fig. 5. Page d'ouverture, L'Art pré-roman
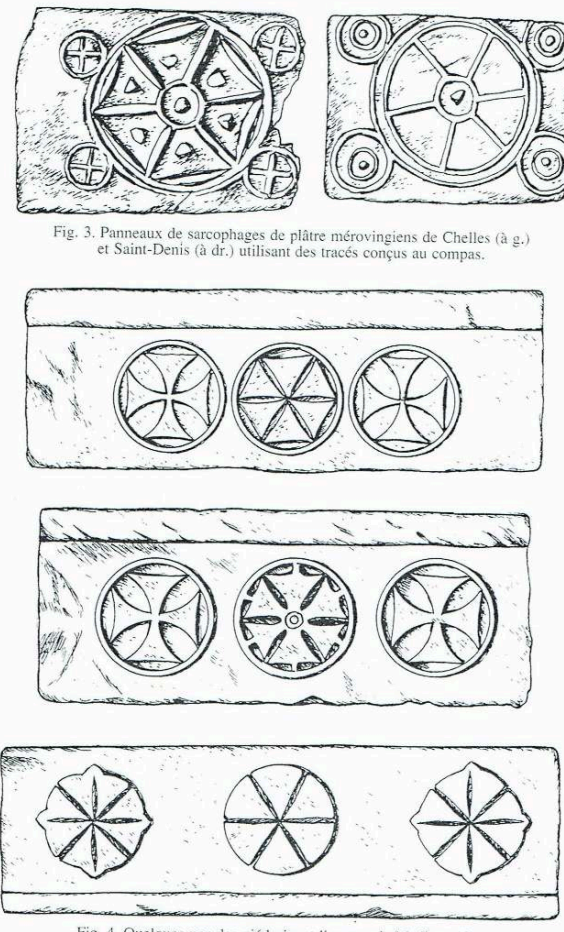

Fig. 4. Quclques-uns des piédroils et linteaux de Medinet $A$ a
utilisant eux aussi des décors faisant appel au compan.

Page d'ouverture, au sein de l'ouvrage de Jean Hubert publié en 1974 aux éditions Chartres.

6 Tous ces grands anciens avaient-ils conscience de l'extraordinaire mouvement de curiosité et de recherche qu'ils mettaient en œuvre ? Peut-être pas, mais ce qui importe c'est que ce mouvement perdure et qu'il nous réunisse aujourd'hui pour nous projeter dans l'avenir. Ce courant réactivé aura des prolongements dans des domaines nouveaux, tels ceux qu'ouvre l'informatique avec notamment le dessin assisté par informatique et d'autres techniques. Adepte pour ma part des relevés graphiques sur papier, avec un instrument de mesure et un crayon, même si d'autres systèmes d'enregistrement d'image existent désormais, telle la lasergrammétrie, je souhaite simplement que les centaines de relevés, de sarcophages, par exemple, que des dizaines de chercheurs ont patiemment collectés, trouvent encore un peu d'utilité en complétant - pourquoi pas? - les précieuses archives dont le Louvre assume la conservation.

7 C'est par ce souhait que je j'achève ce chapitre nostalgique et vous invite à tourner vos regards vers la poursuite du vaste inventaire numérisé des monuments sculptés du IV au $\mathrm{X}^{\mathrm{e}}$ siècle, que nous appelons de nos vœux et dont il faut souhaiter que l'accès soit le plus aisé possible pour tous les chercheurs, tant nationaux qu'étrangers. Car les clivages induits par les frontières tendront à s'effacer au fil des décennies et l'on découvrira ou l'on redécouvrira que les influences artistiques et iconographiques peuvent s'exercer sur de longues distances et dans des durées assez étendues.

$8 \mathrm{Au}$ cours de ces deux Journées d'étude, diverses formes d'expression de l'art de la sculpture ont été présentées mais il en est quelques-unes qui, même si elles ne relèvent pas des formes majeures de cet art, ne doivent pas pour autant être négligées, ce sont les œuvres issues du moulage. Certes, elles sont présentes dans les volumes déjà publiés du Recueil général des monuments sculptés en France pendant le haut Moyen Âge. MM. Marcel 
Durliat, Christophe Deroo et Maurice Scelles, dans le volume consacré à la HauteGaronne, ont pris grand soin d'inventorier tous les fragments d'antéfixes à décor chrétien mis au jour sur le site de Saint-Bertrand-de-Comminges.

Fig. 6. Fragments d'antéfixes
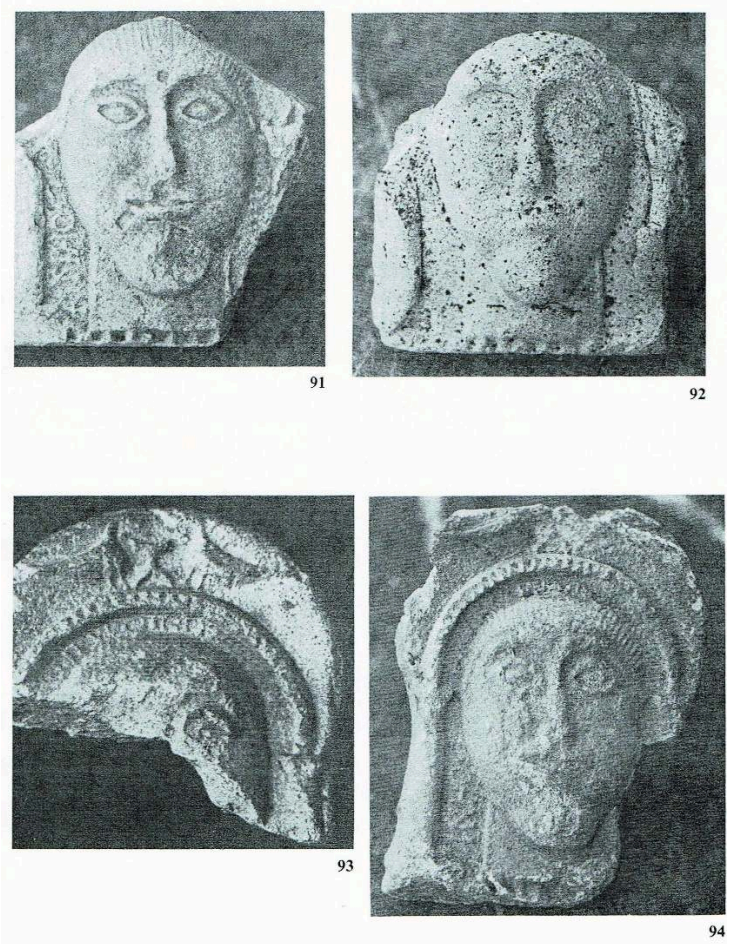

Fragments d'antéfixes, Saint-Bertrand de Comminges, Musée de Comminges.

(c) Chr. Deroo

De même, dans le volume sur Paris, D. Fossard, M. Vieillard-Troïekouroff et É. Chatel ont su accorder toute l'importance qu'ils méritent aux sarcophages de plâtre moulés et exhumés du sous-sol parisien au XIX siècle par Théodore Vacquer. Pour les départements des Yvelines et du Val-d'Oise, la même démarche a été observée par Jacques Sirat, May Vieillard-Troiekouroff et Élisabeth Chatel. Ce n'est pas sur l'inventaire de ce type de monuments que portent mes craintes mais plutôt sur la relative rareté des études qui leur sont consacrées, notamment en ce qui concerne leurs décors. Si certains de leurs thèmes iconographiques, répétitifs, peuvent sembler d'une certaine banalité, gardons-nous d'en négliger l'étude. Il en est qui peuvent s'avérer d'une richesse singulière, telles ces croix inscrites dans un cercle, cantonnées de quatre petits cercles. Et que dire des représentations anthropomorphes, peut-être une représentation du Christ, et de l'association d'une face humaine avec une croix, ou encore d'un décor épigraphique sur une croix. Les recherches stylistiques et morphologiques ont certes leur importance, celles relatives à l'iconographie en ont tout autant, surtout pour la connaissance des mentalités.

10 Le plâtre n'est pas le seul matériau de moulage de monuments, sans doute conviendrat-il aussi d'accorder l'attention qu'ils méritent au stuc et aux décors qui l'utilisent.

11 Un dernier point sur lequel je souhaite attirer l'attention à propos des produits issus du moulage, ce sont les petits objets, telles les ampoules. 

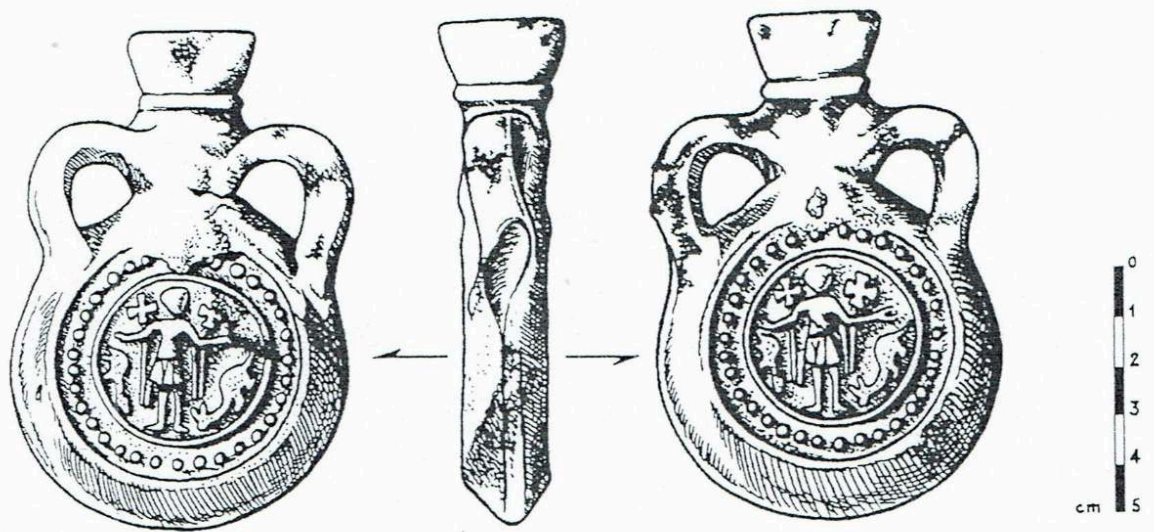

Ampoule de Saint-Ménas de Montereau-fault-Yonne (Seine-et-Marne).

(c) Dessin G.-R. Delahaye

Fig. 8. Ampoule de Saint-Ménas

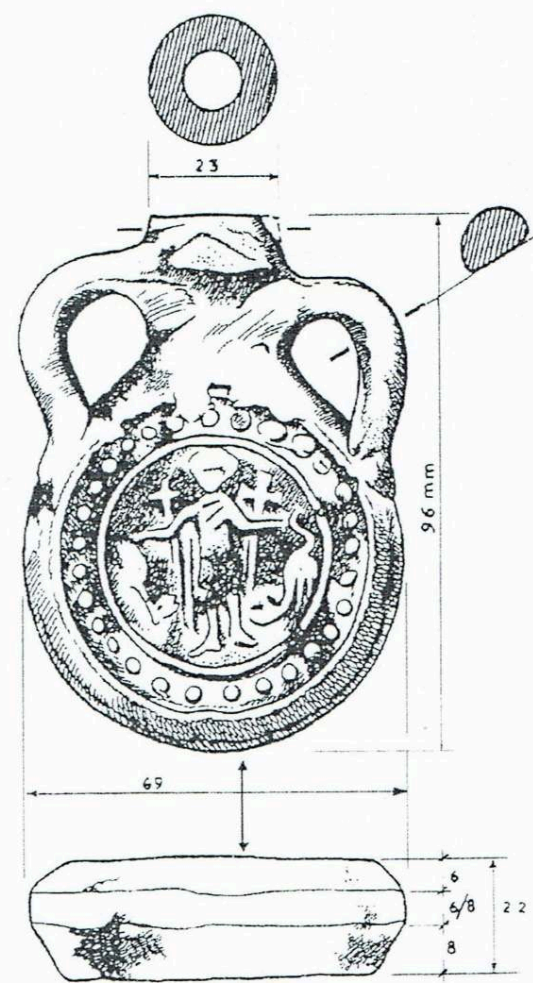

Ampoule de Saint-Ménas de Thorigny-sur-Marne (Seine-et-Marne)

(c) Dessin G.-R. Delahaye 

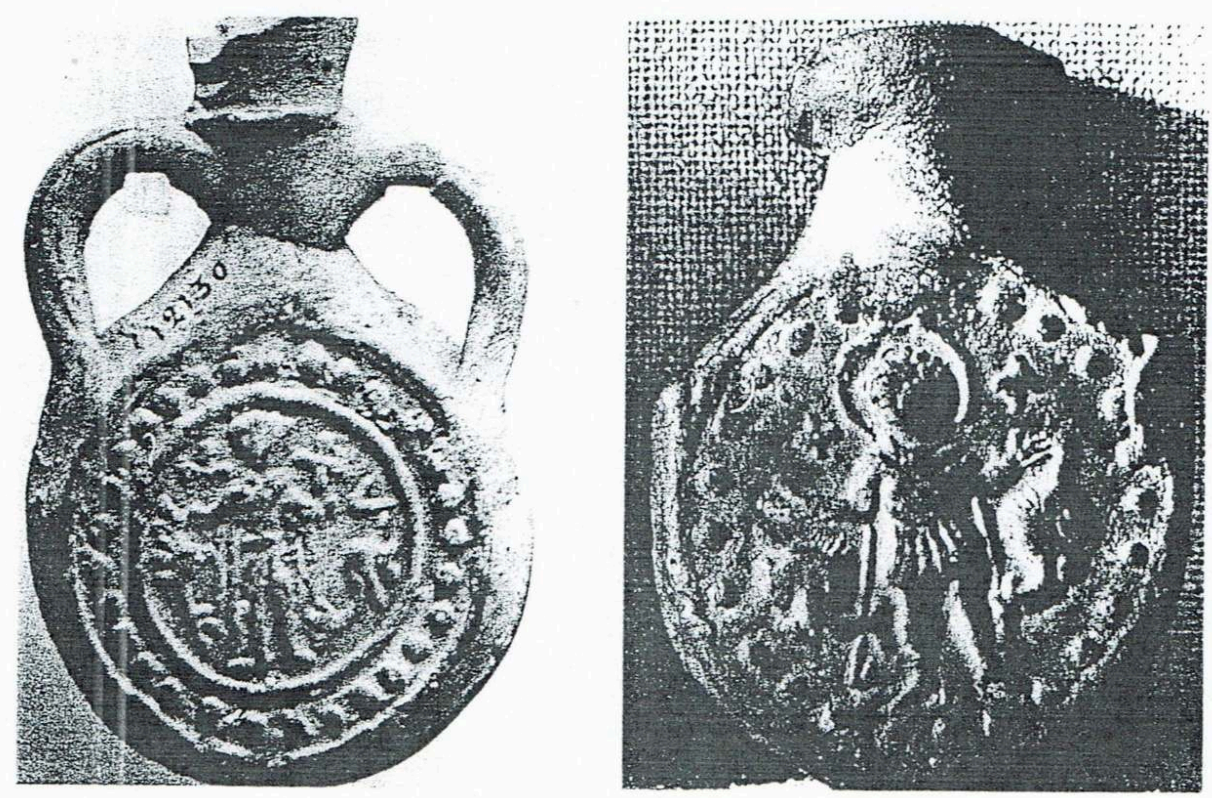

Ampoule de Saint-Ménas conservée au Musée des Beaux-arts et d'archéologie de Genève (Suisse) et ampoule de Saint-Ménas conservée au Musée archéologique de Lausanne (Suisse).

(c) J.-P. Laporte

Bien sûr, leur taille modeste ne peut les faire qualifier de «monuments » et, de ce fait, ces objets ne semblent pas jusqu'ici avoir retenu l'attention des auteurs du Recueil général des monuments sculptés en France pendant le haut Moyen Âge. Pourtant, ils le mériteraient car, outre qu'ils sont parfois les témoignages d'échanges trans-régionaux, ils montrent une variété de décors, notamment épigraphiques, dont on peut s'étonner qu'ils n'aient pas davantage attiré l'attention. Peut-être sera-ce le cas dorénavant grâce aux spécialistes rassemblés ici.

\section{Christian Sapin}

Merci de ces rappels, de ces précisions, de cette mémoire des travaux réalisés dès les années 1970, dans lesquels vous avez effectivement accompli une œuvre à l'époque tout à fait novatrice. Autour de cette question du corpus et de ses limites, ce que vous avez montré en dernier, avec notamment les ampoules de Saint-Ménas, fait partie des questions que l'on se pose de ce que l'on peut associer ou pas à l'intérieur d'un corpus comme le nôtre. Je donne la parole à Fanny Hamonic, conservatrice au Musée d'archéologie nationale, qui va peut-être revenir sur d'autres aspects concernant les limites de ce corpus et la présentation des œuvres.

\section{Fanny Hamonic}

15 Je vais aborder peut-être une perspective plus muséographique, mais qui pose des questions liées aux problèmes d'enjeux et de limites du corpus. Quand j'ai pris mes fonctions au musée d'Archéologie nationale, c'est-à-dire il y a trois ans, l'une de mes premières missions - qui était probablement une légère forme de bizutage - $\mathrm{a}$ été de 
mener à bien le récolement d'un certain nombre d'éléments lapidaires conservés à différents endroits du château : la chapelle, dont je vais vous parler aujourd'hui, mais aussi les sous-sols et les douves. Cela a donné lieu à des séances de travail un peu acrobatiques et poussiéreuses. Je me suis rapidement rendu compte de l'intérêt de cette collection, mais aussi de sa dispersion au sein des espaces, voire parfois, il faut le reconnaître, de son absence de réelle prise en charge. Mon intégration au réseau SATHMA a été l'occasion de revenir un peu sur l'histoire de cette collection et, plus particulièrement, sur ce qu'on appelle le "musée chrétien » du musée d'Archéologie nationale. Je vous remercie beaucoup de m'avoir conviée à cette table ronde qui est davantage un point de départ dans ma recherche qu'un aboutissement et j'espère que nos échanges vont m'aider à avancer. Les pistes sur lesquelles je me suis lancée entrent déjà en résonance avec ce qui a été dit par Pierre Yves Le Pogam, sur la réception moderne de cet art sculpté ${ }^{\text {. }}$

Fig. 10 Musée chrétien dans la chapelle du château de Saint-Germain-en-Laye

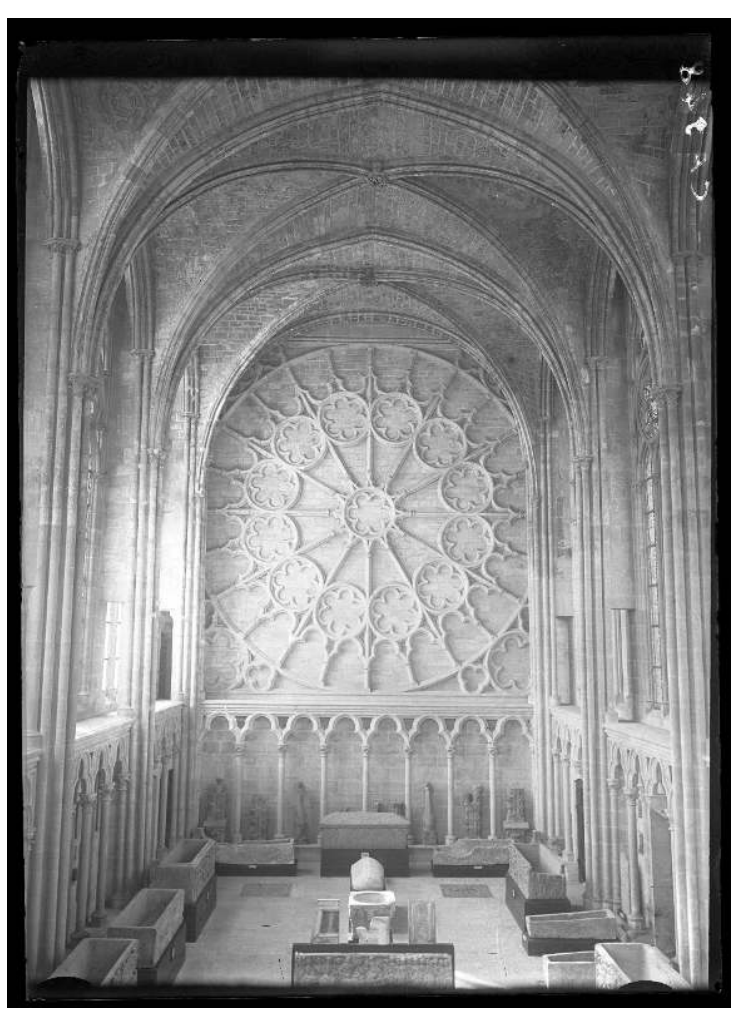

Musée des Antiquités nationales, vue du côté de la rosace. Plaque de verre au gélatino-bromure d'argent, format 13 x 18, PV_13x18_01394, ancien cliché CF948. Numérisation et retouches par Loïc Hamon, centre des archives.

(c) MAN

16 La collection qu'on désigne sous le nom de "musée chrétien » au musée d'Archéologie nationale (ci-après MAN) désigne un ensemble de sculptures, de sarcophages, de stèles et de moulages qui autrefois était présenté dans la chapelle du château afin d'évoquer, selon les mots de Salomon Reinach, « l'histoire du christianisme dans la Gaule romaine et mérovingienne ${ }^{2} »$. Vous avez un exemple en image de ces anciennes présentations et je vais vous expliquer comment elles ont évolué.

Le nom de Salomon Reinach est très fortement attaché à celui du musée chrétien et, en débutant mes recherches, je pensais, comme la plupart de mes collègues, qu'il en était 
l'instigateur, évidemment parce qu'il est conservateur du MAN à ce moment-là, mais aussi parce qu'il publie le premier guide de visite consacré à cet espace, en $1903^{3}$. En fait, de rapides recherches menées dans les archives du MAN m'ont permis de me rendre compte qu'en réalité, le projet d'un musée chrétien existe pratiquement dès l'origine du musée (ouvert en 1867), puisqu'en 1873 on trouve déjà dans le Journal Officiel de la République Française une mention qui dit: "c'est dans cette chapelle du château de Saint-Germain-en-Laye que l'on doit installer les collections archéologiques relatives aux origines du christianisme dans la Gaule, après qu'on l'aura débarrassée des horribles badigeons dont elle a été couverte par les artistes au service de Louis XIV $»^{4}$. On parle bien de peintures d'Aubin Vouet !... Il paraît logique qu'on ait très tôt envisagé l'usage futur de la chapelle, tout simplement parce que, dès le début de la restauration du château, on nous dit bien, et c'est une évidence, que c'est un édifice de première importance. Pourquoi, alors, ce "retard» entre l'ouverture du musée et l'ouverture de la chapelle? Tout simplement parce que les restaurations du bâtiment, conduites par Eugène Millet, se poursuivent pour l'intérieur de la chapelle jusqu'en 1900.

18 L'espace est donc ouvert au public à partir de 1902 et permet de présenter, auprès de quelques originaux, mais pas tant que cela, de nombreux moulages qui proviennent de collections et d'échanges, mais aussi des moulages réalisés par Benoît-Claude Champion, chef de l'atelier de moulage du musée à l'époque ${ }^{5}$. Ces moulages sont réalisés spécialement à la demande de Salomon Reinach, pour constituer un ensemble plus cohérent pour le musée chrétien. Dès le départ, l'espace est conçu par Reinach comme un corpus d'œuvres destinées à illustrer les débuts du christianisme et choisies surtout pour leur iconographie, la présence d'inscription ou de symboles explicites par rapport à ce sujet. À ce titre, on comprend très bien l'importance qu'il va accorder aux moulages, qui permettent d'élargir le corpus et d'accentuer sa pertinence. Aujourd'hui, dans le cadre de notre corpus SATHMA, il est vrai que les moulages peuvent sembler un peu en marge de la réflexion; cependant ils y apparaissent, quand ils ont valeur d'originaux ou même quand ils peuvent documenter un ancien état de conservation. Je ne cite qu'un seul exemple, dans le tome 3 du Recueil général des monuments sculptés, où l'on évoque un sarcophage avec inscription de Tournedos-sur-Eure, moulage conservé au MAN, à l'origine un objet du musée de Louviers, qui a disparu ${ }^{6}$. Je m'interroge sur la place qu'on pourrait accorder au moulage dans le cadre du corpus. Le corpus va apporter beaucoup dans le réexamen de nos moulages $^{7}$, pour savoir par exemple si certains ont valeur d'originaux par rapport à des œuvres conservées dans d'autres musées de France.

Pour continuer sur l'histoire de ce musée chrétien dont on peut voir l'un des premiers états sur la figure 10, l'espace va rencontrer, dès son ouverture, un succès très important et Reinach dit dès 1903 qu'il a obtenu pour la chapelle une autorisation spéciale d'ouverture en dehors des horaires classiques de visite ${ }^{8}$. Pourtant la suite de l'histoire est assez houleuse puisque l'espace subit un réaménagement entre 1931 et 1935 , puis va rouvrir - il y a peu d'informations sur toute cette période - avant que, très rapidement, la partie centrale de l'espace soit dégagée et que les éléments les plus volumineux soient rangés le long des murs pour permettre la tenue d'événements : c'est ce que l'on peut voir sur ces images (fig. 11 et 12). On sait notamment que la Callas s'est produite dans la chapelle du MAN. En 1964, les sarcophages sont toujours, d'après un document conservé aux Archives nationales, «entreposés dans la chapelle »; c'est sans équivoque, il ne s'agit plus de muséographie à proprement parler. Ensuite, d'après 
différents collègues, qui sont mieux informés que moi, c'est au moment de la restructuration du musée, après 1974, que des éléments encore présents au sol ont été évacués de l'espace, et que l'on n'a plus conservé que ce qui était présenté aux murs. Il est encore assez difficile de comprendre exactement pourquoi.

Fig. 11 Chapelle, rose ouest aveuglée

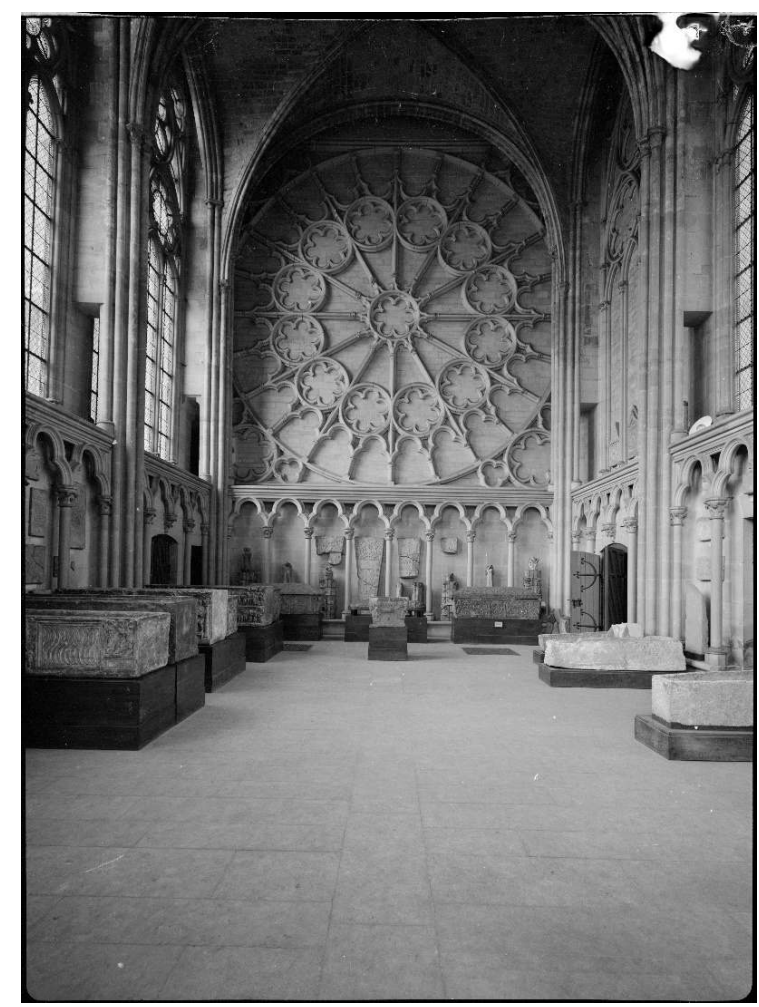

Branner, 1963. Négatif noir et blanc, gélatino-bromure, support pellicule, n63S01756. Ministère de la Culture (France), Médiathèque de l'architecture et du patrimoine, diffusion RMN-GP. 
Fig. 12 Intérieur de la chapelle, ensemble vers le nord-est

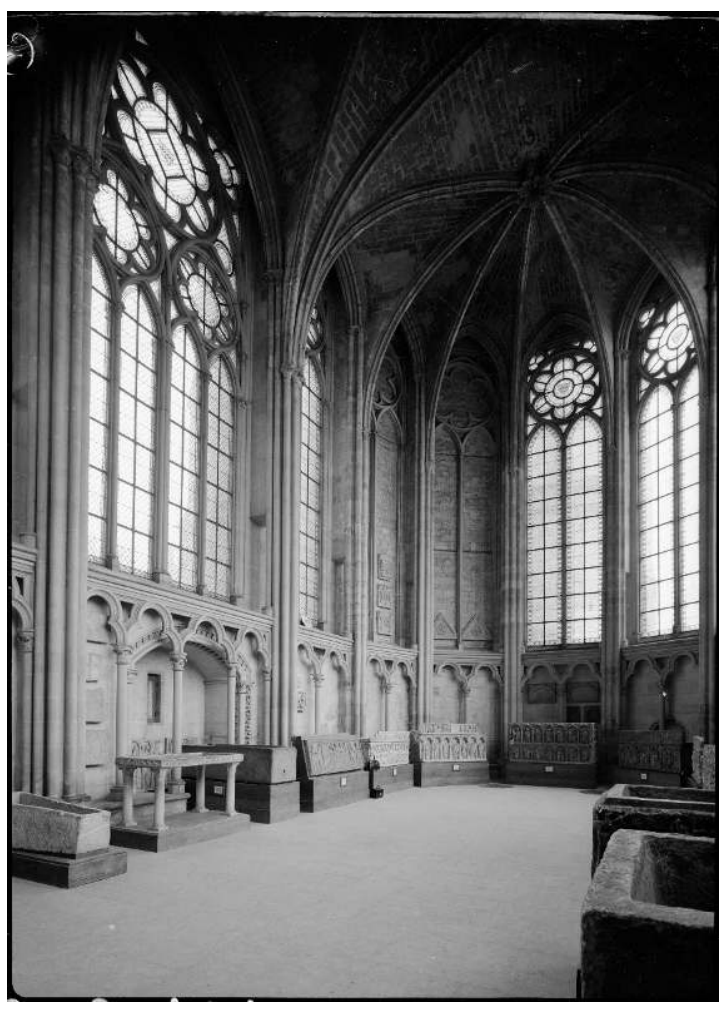

Branner, 1963. Négatif noir et blanc, gélatino-bromure, support pellicule, n63S01754. Ministère de la Culture (France), Médiathèque de l'architecture et du patrimoine, diffusion RMN-GP.

Je voudrais dire que les vicissitudes du musée chrétien de Saint-Germain n'ont pas manqué de m'évoquer celles de la section des antiquités chrétiennes du musée du Louvre. La situation y est encore plus complexe puisqu'il s'agit d'une section déjà envisagée par Jeanron, dont l'idée sera reprise sans beaucoup plus de succès par Laborde ${ }^{9}$; finalement elle va renaître de ses cendres de manière officieuse avec Barbet de Jouy $^{10}$. Cette initiative, qualifiée dans l'Histoire du Louvre, d'«originale et controversée ${ }^{11}$, aboutit avec Étienne Coche de La Ferté en 1954, mais dans des conditions extrêmement délicates, puisque La Ferté est placé sous le contrôle des conservateurs en chef des autres départements, qu'il manque beaucoup d'espace et que, bien sûr, les départements d'origine des différentes pièces sont assez peu enclins à les céder. On comprend bien qu'au Louvre, ce qui a posé problème, c'est la transversalité du projet et il me semble que c'est l'une des différences importantes entre cette section et celle du MAN, puisque cette dernière déjà ne suscitait pas de rivalités dans une conservation qui était assez restreinte et qui, par ailleurs, avait été voulue quasiment dès la création du musée. Il me semble que cela rapproche en fait le MAN du cas du département des arts de l'Islam, qui est un département qu'on cite souvent en contrepoint de cette section des antiquités chrétiennes mais qui, à ma connaissance, a bénéficié dès le début d'un inventaire dédié dressé parallèlement à celui du département des Objets d'art ${ }^{12}$. Je ne vais pas revenir sur les débats récents autour du département des arts de Byzance et des chrétientés d'Orient, mais on pourrait en parler, d'autant que j'ai cru comprendre que le projet reprenait. En tout cas, force est de constater que ces musées chrétiens ont eu du mal à s'épanouir dans 
nos musées respectifs, et je pense que cette histoire mériterait qu'on s'y attache un peu plus avant.

Pour conclure, j'aimerais revenir à Salomon Reinach parce que, finalement, la problématique du cloisonnement est au cœur de l'histoire de ces musées chrétiens, et Reinach, justement, à son époque, encourageait le décloisonnement de la recherche. Il faisait preuve d'un véritable intérêt pour les corpus et pour les démarches comparatives; et je pense que le cadre de notre corpus SATHMA s'inscrit dans cette belle lignée qui je l'espère, permettra au MAN de mieux documenter l'histoire de ses collections et de la muséographie de la chapelle. J'aimerais, une fois que ce travail scientifique sera réalisé, trouver l'opportunité de peut-être faire renaître de ses cendres ce musée chrétien, notamment grâce à des prises de vues 3D de l'espace, des prises de vues des œuvres qui nous permettraient de reconstituer, en réalité augmentée, la présentation de Salomon Reinach et de proposer à nos visiteurs une visite originale de cet espace en les transposant au début du XXe siècle. Il me semble que ce serait un bel aboutissement de cet important travail et une vraie valeur ajoutée à la visite de la chapelle. (a problématique de la matérialité des œuvres, et plus précisément de la provenance du matériau. Il est bien évident, à mon humble avis, et je pense que vous en êtes convaincus, que l'identification de la nature du matériau en œuvre dans la sculpture est un indice à ajouter et présente de l'intérêt par rapport à la mise en ligne d'un corpus. Bien sûr cela demande un travail analytique assez énorme et il n'est pas dit que ce soit réalisable de façon immédiate. Pourtant, la connaissance de la nature de la pierre en œuvre et de sa provenance est un indicateur précieux de l'histoire de l'œuvre, à plusieurs niveaux, et on a bien vu à travers les différentes présentations de ce colloque qu'on ne pouvait pas appliquer un seul schéma. Je retiendrai la conclusion de Monsieur Gregor qui était qu'il y avait une adaptation de l'exécutant aux matériaux. Je dirais presque que c'est une question d'opportunisme, parce que, ce que l'on a vu aussi transparaitre, hormis pour le cas particulier des sarcophages, c'est qu'on a dans 
l'ensemble de ces œuvres beaucoup de réemplois, voire de réemplois de réemplois avec, à nouveau, probablement pas un seul schéma à retenir mais une multitude de schémas adaptés à des questions d'opportunité et de saisie de l'opportunité. L'approvisionnement des chantiers de construction et la réalisation d'œuvres à destination architecturale, telles que des chapiteaux ou un chancel, se faisait à partir d'édifices existants à proximité, qui étaient la ressource carrière. Ce n'est pas pour autant qu'il n'y avait pas de savoir-faire d'extraction; c'est-à-dire qu'on utilise en réemploi des matériaux parce qu'ils sont disponibles et non pas parce qu'on ne sait pas les extraire, puisqu'on voit bien que, pour les sarcophages, il y avait un grand savoirfaire de l'extraction. Et puis il y a aussi des choix de matériaux à destinées précises : citons par exemple la commande de colonnes en marbre destinées à la réalisation d'un tombeau ou d'un ouvrage dans un édifice. On peut s'interroger sur le fait de faire venir ces colonnes de loin en raison de la nature du matériau ou de la forme. Je dirais que très souvent ce qui transparaît c'est que l'on a souhaité la forme en priorité, mais peut-être pas toujours, et à nouveau il ne faut pas appliquer un schéma unique. Quand on regarde la crypte de Jouarre, par exemple, on voit bien des colonnes qui sont utilisées pour leur forme et leur valeur architecturale, avec des natures de marbre très différentes et un rendu hétérogène. A contrario, toujours à Jouarre, il y a une plus grande homogénéité au niveau du rendu des chapiteaux, avec pourtant au moins deux fournitures différentes de marbres et peut-être des lieux d'approvisionnement différents. Dans cet édifice, la mise en œuvre romane, du XII ${ }^{e}$ siècle, réemploie des chapiteaux du haut Moyen Âge, dont on peut se demander s'ils étaient en œuvre, à cette époque, dans un même édifice. Est-ce que la provenance initiale est un édifice antique avec une resculpture de chapiteaux antiques? On aurait donc des re-sculptures à l'époque romane de chapiteaux du haut Moyen Âge qui étaient peut-être originellement des chapiteaux antiques... Et là, se pose une question: pourquoi sculpter un chapiteau dans un chapiteau? Pourquoi ne pas sculpter un chapiteau dans un autre bloc, ce qui apporterait moins de contraintes? Car utiliser un chapiteau préalable pour faire un chapiteau impose une contrainte formelle. Il subsiste donc nombre de questions, et une des réponses est finalement donnée dans la conclusion de Monsieur Gregor : il y a certainement beaucoup d'opportunisme.

\section{Christian Sapin}

Merci de rappeler cette question du réemploi qu'on avait abordé justement dans le cas du corpus. L'enregistrement est bien notifié dans les notices pour ces réemplois avec les questions que vous soulevez pour les chapiteaux: s'agit-il de réutilisation de l'édifice précédent ou de récupération dans des villae? On voit qu'il y a des périodes plus propices à cela, notamment à l'époque carolingienne durant laquelle un certain nombre de chapiteaux de marbre sont réutilisés. Ce qui est intéressant est bien entendu la signification donnée au marbre, à ce riche matériau souvent remployé pour marquer l'ancienneté du lieu restauré, agrandi ou reconstruit. Cela nous permet de faire la transition avec le monument lui-même. François Héber-Suffrin connait justement bien la sculpture et les monuments du haut Moyen Âge et peut nous parler de l'intérêt de cette question pour le corpus, qui ne doit pas simplement accumuler des notices d'œuvres ou des matériaux, mais aussi, à travers les œuvres, donner sens aux monuments. 


\section{François Héber-Suffrin}

27 Je voudrais rendre hommage, à mon tour, à May Vieillard-Troiekouroff et à Élisabeth Chatel et indiquer que je suis ravi de prendre part au colloque SATHMA pour traiter des questions d'histoire de l'art et notamment de l'art monumental carolingien. Elles ont été succinctement abordées, excepté par mon camarade Christian Sapin qui a montré des pièces sculptées carolingiennes disparates, trouvées en fouilles, et appartenant de fait à des contextes peu reconnaissables. Pour ma part, je voudrais, selon le point de vue de l'historien de l'art, éclairer le problème spécifique posé par le chapiteau carolingien. Cela oblige naturellement à se tourner vers l'Allemagne où se situent la plupart des grandes réalisations carolingiennes en matière de chapiteaux. Je voudrais insister sur la façon dont les Carolingiens, dans le cadre de la «Renaissance carolingienne ", se réfèrent de manière assez délibérée à l'Antiquité. Il faut remarquer, en étudiant les chapiteaux d'un côté ou de l'autre du Rhin, que les sources ne sont pas strictement les mêmes. En effet, en France, dans une zone en grande partie non germanique et qui a été longuement et profondément romanisée, les modèles des chapiteaux carolingiens sont probablement des pièces locales déjà très souvent et très fortement déformées ou simplifiées par rapport au chapiteau canonique. J'en prends pour exemple la sculpture de l'église de Germigny-des-Prés dans le Loiret, sur laquelle j'ai récemment travaillé1 ${ }^{13}$, où les modèles des chapiteaux ont déjà connu les déformations héritées de toutes les transformations successives de la basse Antiquité. Dans leur traitement, les chapiteaux de Germigny-des-Prés sont très caractéristiques des pratiques carolingiennes: imiter le chapiteau antique à travers les témoins architecturaux conservés. Sur les chapiteaux de Germigny, la palmette remplace très systématiquement la feuille d'acanthe comme on le trouvait déjà, sous une forme plus ou moins simplifiée, sur des chapiteaux probablement tardo-antiques en marbre de l'Ouest de la France (par exemple un chapiteau à palmettes remployé au VII siècle dans le baptistère de Poitiers). À l'appellation « chapiteau corinthien » hérité de l'Antiquité, on devrait peut-être plutôt substituer " chapiteau corinthisant », car si l'on reconnaît, comme sur les chapiteaux qui encadrent l'abside de Germigny, la collerette propre au modèle corinthien, on assiste à une simplification géométrique de la partie supérieure. Ainsi on comprend, à travers l'analyse de ces chapiteaux, que l'on a adopté des schémas existants déjà très simplifiés. En revanche, du côté germanique, dans des zones qui n'ont été colonisées que tardivement et qui n'avaient, pour la plupart, jamais été colonisées par les Romains, on trouve, au contraire, des schémas qui renvoient à des modèles beaucoup plus classiques, sans doute à partir des apports venant d'Italie à l'époque de Charlemagne et de ses successeurs... Ces derniers chapiteaux sont donc beaucoup plus proches des modèles canoniques. Il sera important, dans le cadre du corpus SATHMA, de penser à se référer aussi aux grands exemples carolingiens, comme ceux de Fulda (la cathédrale ou l'église Saint-Michel) ou encore de Corvey, dont on possède un reflet assez différent du côté français.

Je voudrais également revenir sur la question des chapiteaux simplement épannelés et qui ont pu être peints à l'origine. Il faut rappeler l'existence, au rez-de-chaussée du Westwerk de Corvey, au sommet des quatre piliers qui soutiennent le sol de la chapelle supérieure, de chapiteaux corinthiens très bien formulés dont les détails n'ont été gravés que sur certaines volutes. Or, la chercheuse Hilde Claussen a remarqué qu'il subsistait, dans les pores de la pierre, de la couleur ${ }^{14}$ : on peut donc penser, et c'est une 
hypothèse qui a été formulée, que la gravure sur certaines des feuilles est en fait un modèle à suivre par la peinture.

Le dernier point sur lequel je voudrais insister - pour revenir sur les sculptures disparates, et peut-être carolingiennes, présentées par Christian Sapin - c'est la nécessité de réunir, dans le cadre du corpus, un répertoire relativement important de pièces. C'est en effet à cette seule condition que l'on pourra véritablement mettre en relation des chapiteaux encore en place et bien datés, comme pour Germigny, avec des chapiteaux qui sont dispersés et sans contexte. On pourra alors se référer à ces exemples datés pour proposer des rapprochements, comme dans le cas de la palmette des chapiteaux de Germigny, figurant également sur ceux de Flavigny-sur-Ozerain (Côte-d'Or), dans une forme certes plus simplifiée mais dans un contexte qui paraît semblable. Il faudra donc produire un corpus élargi de manière à ce que l'historien de l'art y trouve son compte.

\section{Christian Sapin}

Merci pour ce rappel des spécificités carolingiennes et cet hommage auquel on s'associe tous. Tu nous dis, à juste titre, que pour les chapiteaux, il ne faut pas oublier de se référer aux monuments ayant conservé encore leur sculpture, notamment en Allemagne. Que t'inspire un autre type de sculpture, celle du mobilier liturgique dont on a parlé notamment avec Pascale Chevalier et les exemples de l'Antiquité tardive? Est-ce qu'il n'y a pas une différence d'approche à avoir sur ce décor, comme celui d'entrelacs typiques, souvent considérés comme appartenant à un chancel. Est-ce que là il n'y a pas justement des pistes de réflexion à développer comme l'ont fait nos collègues suisses récemment?

\section{François Héber-Suffrin}

Oui certainement mais dans le cas du mobilier liturgique, c'est encore plus difficile, car il faudrait être certain que les fragments que l'on trouve soient carolingiens. Bien souvent, et l'exemple du chancel de Saint-Pierre-aux-Nonnains de Metz, pour lequel les datations varient, le montre, on est incapable de dire s'il présente, avec des styles différents, une certaine homogénéité ou pas, s'il est composé de pièces à la chronologie différente ou non et si certaines plaques, comme celle du Christ, doivent être complètement détachées de l'ensemble, bien que les techniques de sculpture soient semblables. C'est très difficile à dire... 

particulier à la limite de l'enregistrement des fragments que nous trouvons. Pour les sarcophages, par exemple, quand on regarde les réserves des musées mais surtout toutes ces fiches qui ont été remplies par les trois chercheuses du Recueil dans les années 50,60, 70, la place des sarcophages et des fragments sont importants. Emmanuelle Boube a évoqué lors de ce colloque l'intérêt qu'elle portait aussi en tant que chercheuse au recensement des sarcophages lisses et sans décor. Fabrice Henrion a bien montré également comment on pouvait admettre qu'un certain nombre de sarcophages avec des stries soit retenu, ce que l'on a fait dans le cadre du corpus. Mais on peut aussi se poser la question sur les différences entre, d'une part, l'intérêt du chercheur pour un enregistrement total des éléments appartenant à ce domaine funéraire (même si certains, notamment quand il s'agit de plaques complètement lisses, nous posent un problème, puisque l'identification en tant que sarcophage n'est pas si évidente) et, d'autre part, le fait que les limites de la description peuvent évidemment être très vite atteintes. Je parle de plaques, mais ça peut être aussi un fragment dont on ne sait pas très bien s'il est important ou pas. Le fait qu'il soit en marbre, évidemment, nous incite à penser qu'on a affaire à un élément de décor ou à un élément architectonique, mais cela reste un exercice difficile.

Il y aussi la question des musées. Fabrice Henrion a évoqué, je crois, le problème du stockage. En effet, nous avons dit tout l'intérêt que nous portions à ces fragments, mais quand on discute avec nos amis conservateurs de musée, ils lèvent souvent les yeux au ciel quand il s'agit de stocker, d'engranger, de présenter des dizaines de sarcophages. Et je dois dire que, pour ma part, maintenant je suis partisan, quand on fouille et quand les sarcophages n'ont pas un caractère tout à fait exceptionnel ou qu'ils ne sont pas menacés par un aménagement, de laisser les sarcophages in situ, car quelquefois c'est encore le meilleur moyen de les conserver.

41 Je pense que vous en serez remercié par un certain nombre de responsables de musées... Au MAN, on n'a pas une collection envahissante au point d'en être devenue ingérable mais, déjà, malgré le caractère assez restreint de cette collection, les conditions de stockage ne sont pas forcément idéales, parce que, longtemps, on s'est peu intéressé à ces sarcophages qui ne présentaient pas de décor particulier et qui sont donc souvent restés en extérieur. Vous pouvez imaginer ce que cela suppose en termes d'altérations. Et puis aussi, malheureusement, en ayant été parfois déplacés et redéplacés, les sarcophages fragmentaires ont été un peu dispersés ou abîmés. Le numéro d'inventaire qui n'apparaît que sur une partie du sarcophage peut avoir été perdu, il faut recoller les fragments et organiser un puzzle géant avec des œuvres lourdes et de grandes dimensions. Cela suppose un très gros chantier de collections, qui se consacre uniquement aux sarcophages, et donc des moyens et de l'espace. C'est un sujet important et intéressant et je dois dire que pour le MAN, il faudrait en effet consacrer un chantier de collections uniquement aux sarcophages pour savoir où on en est, car on a perdu la trace de certains éléments.

\section{Christian Sapin}

À propos aussi d'autres éléments dont on n'a pas parlé que sont les moulures et les bases, qui peuvent être nombreux, on peut citer le choix fait à Genève dans la présentation du sous-sol archéologique de la cathédrale : l'ensemble du lapidaire y est placé, derrière des grilles, pour montrer la quantité que ça représente, pour que l'on se 
rende compte quelle masse cela constitue, car quelques fois la pièce n'est pas exceptionnelle, mais peut aussi être intéressante. Il y a donc plusieurs attitudes à avoir. On peut aborder également la question des matériaux qui se conservent plus ou moins bien, on a parlé des stucs, et des éléments en plâtre dont il faut prendre soin. retrouvent d'une région à l'autre ou d'un pays à l'autre. Donc, aujourd'hui, plus ces corpus sont larges, notamment à l'échelle européenne, plus cela permettra de percevoir justement, peut-être, ces circulations. J'avais été frappé, pour ma part, en travaillant sur la Bourgogne, de voir, et cela a été évoqué par Anne-Bénédicte Mérel-Brandenburg, que certains chapiteaux qu'on a pu identifier étaient effectivement en marbre de Bourgogne alors qu'en même temps, leurs formes étaient identiques à celle des chapiteaux des Pyrénées. Donc une fois qu'on a dit que le marbre était local, est-ce que le sculpteur venait d'ailleurs ou est-ce qu'il avait des carnets de modèles, comme on le dit souvent notamment pour les époques ultérieures? Cela fait partie des questions qui sont encore très ouvertes, pour lesquelles, on peut l'espérer, le corpus, en engrangeant beaucoup de données accumulées sur les origines des œuvres, sur leur présence dans tel ou tel monument, pourra nous permettre de cartographier des informations. À partir des données issues du corpus, nous pourrons en effet dresser facilement des cartes de répartition, pas seulement comme le faisait Jean Hubert à travers des cartes, qui étaient déjà tout à fait nouvelles dans ces années 1950, mais aussi en multipliant les données et en les croisant, notamment à partir des carrières qu'on commence à mieux identifier.

Nous avons donc croisé beaucoup des informations et des ouvertures sur différents aspects. Les 13 volumes anglais en ligne nous permettent déjà d'aborder un ensemble de questions. Pour l'Italie on le fait depuis longtemps à partir des volumes papier. Et pour la France, on dispose de quelques volumes publiés par le Comité des travaux historiques et scientifiques (CTHS). Ils avaient été un peu critiqués à une époque, il faut le rappeler, justement parce que la relation avec le monument n'avait pas toujours été faite. C'est ce que l'on voudrait éviter aujourd'hui à travers ce corpus de la sculpture qui s'adosse au corpus européen (ANR Care) sur l'architecture que nous avons lancé il y 
a un certain nombre d'années, Il est loin d'avoir abouti pour la France mais est déjà beaucoup avancé dans d'autres pays. Il permettra de croiser les données sur les œuvres sculptées et sur l'architecture. Nous pouvons nous donner rendez-vous dans quelques années pour faire le point sur toutes ces avancées.

\section{NOTES}

1. Supra, article de P.-Y. Le Pogam.

2. Salomon Reinach, Le musée chrétien dans la chapelle de Saint Louis au château de Saint-Germain-enLaye, Paris, 1903, p. 19.

3. S. Reinach, op. cit. note 2.

4. "Le musée des antiquités nationales de Saint-Germain-en-Laye. Visite de la Société d'Anthropologie ", Journal Officiel de la République Française, 31 octobre 1873, pp. 6640-6641.

5. MAN, centre des archives, correspondance Benoît-Claude Champion, lettre de Benoît-Claude Champion à Salomon Reinach, $1^{\text {er }}$ décembre 1900.

6. Élisabeth Chatel, Jacques Sirat, May Vieillard-Troiekouroff, Recueil général des monuments sculptés en France pendant le haut moyen-âge (IVe-Xe siècles, vol 3, Val-d'Oise, Yvelines, Paris, CTHS, 1984, pp. 77, 101, $n^{\circ} 266$ (nº d'inventaire MAN 34093).

7. Les moulages du MAN sont désormais intégrés à la collection d'histoire de l'archéologie, qui bénéficie depuis la fin de l'année 2020 de l'expertise d'un conservateur spécialement nommé à cet effet, Isabel Bonora-Andujar.

8. S. Reinach, op. cit. note 2, p. 20.

9. Geneviève Bresc-Bautier (sous la dir.), Histoire du Louvre, vol. II, Paris, 2016, p. 143.

10. Ibid., p. 318.

11. Ibid., p. 473.

12. G. Bresc-Bautier (sous la dir.), Histoire du Louvre, vol. III, Paris, 2016, p. 103.

13. François Héber-Suffrin, "Germigny, le décor sculpté : la pierre », Germigny-des-Prés, un nouveau regard, Christian Sapin dir., hors-série $n^{\circ} 11,2019$, du BUCEMA, en ligne (https:// journals.openedition.org/cem/16123)

14. Échange oral avec Mme Claussen; cf. aussi Hilde Claussen, «Vorzeichnungen und Fragmente karolingischer Stuckfiguren : neue Funde im Corveyer Westwerk", Stuck des frühen und hohen Mittelalters. Geschichte, Technologie, Konservierung, Mathias Exner dir., Munich, 1996, pp. 61-71, cf. p. 69.

\section{RÉSUMÉS}

Nous avons pensé que, plutôt qu'une seule conclusion, il était intéressant de proposer cette table ronde permettant de réunir plusieurs participants du colloque SATHMA qui s'est tenu les 24 et 25 Septembre 2020 à l'École du Louvre et dont ont été tirées les contributions précédentes. Fanny 
Hamonic, conservatrice au Musée d'archéologie nationale, Lise Leroux ingénieur de recherche au Laboratoire de recherche des monuments historiques, Gilbert-Robert Delahaye, président honoraire de plusieurs sociétés d'érudition et François Héber-Suffrin, maître de conférences émérite des universités, apporteront ainsi leurs contributions en contrepoint à ce qui a été dit précédemment.

\section{AUTEURS}

\section{GILBERT-ROBERT DELAHAYE}

Président honoraire de plusieurs sociétés d'érudition

\section{FANNY HAMONIC}

Conservatrice au Musée d'archéologie nationale

\section{LISE LEROUX}

Ingénieur de recherche au Laboratoire de recherche des monuments historiques

\section{FRANÇOIS HÉBER-SUFFRIN}

Maître de conférences émérite des universités

\section{CHRISTIAN SAPIN}

Directeur de recherche émérite au CNRS, Umr 6298 Artehis Dijon 